\title{
WATER HAMMER IN A BRANCHED PIPE
}

\section{By Sukeyuki Shima*, C.E. Member and Mikio Hino**, C.E. Member}

Synopsis: This paper presents the experimental and theoretical results of the effect of water hammer in a surge tank or a branch pipe with restricted-orifice, describing the relationships between the transmission coefficients and the orific-earea ratio, and also between the maximum pressure rise and the closure time of gate.

\section{INTRODUCTION :}

In the previous paper ${ }^{1}$, the authors developed a theory dealing with the water hammer phenomena in a restricted-orifice surge tank system, and showed that the transmission coefficient in such a pipe system increases abruptly, for the case of rapid closure of gate or control valve, below a certain value of the orifice-area ratio $A_{p} / A_{0}$ (orifice area/pipe area) which depends on the ratio of the initial flow velocity, $V$, to the wave velocity in the pipe, $\alpha$, and which is far smaller than usually anticipated. The previous study neglected the effect of water hammer in a surge tank. However it has been exclusively investigated in the present study. For convenience of treatment, the surge tank has been replaced with a branch pipe (with the same diameter and made of the same material as the main pipe). An orifice of different sizes has been inserted at the base of the branch pipe, and the relationships between the transmission coefficient and the orifice-area ratio and between the maximum pressure rise and the closure time of gate have been examined through experiments and a theoretical analysis.

\section{Theory of Water Hammer in a Branched Pipe ${ }^{2 \gamma, 3)}$ :}

Let us assume a pipe line system shown in Fig. 1. The flow velocities, wave velocities, total heads, pipe areas and pipe lengths are denoted respectively by $V, \alpha, H, A$ and $L$. Subscripts 1,2 and 3 represent the type of each pipe. The constant head is assumed at points $C$ and $D$.

Fig. 1 Shematic Diagram of Branched Pipe System.

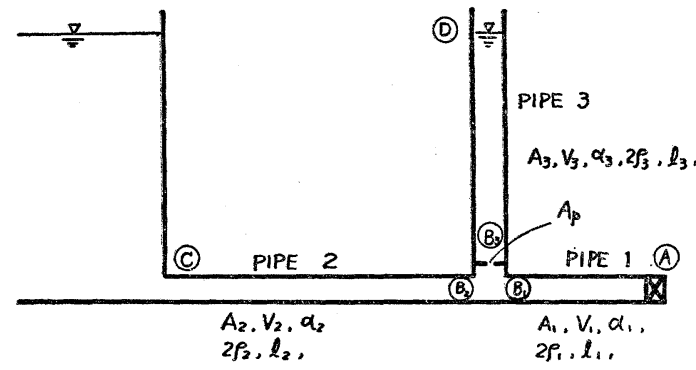

(a) Foundamental equations.

The relationships between $H$ and $V$ are written in a dimensionless form;

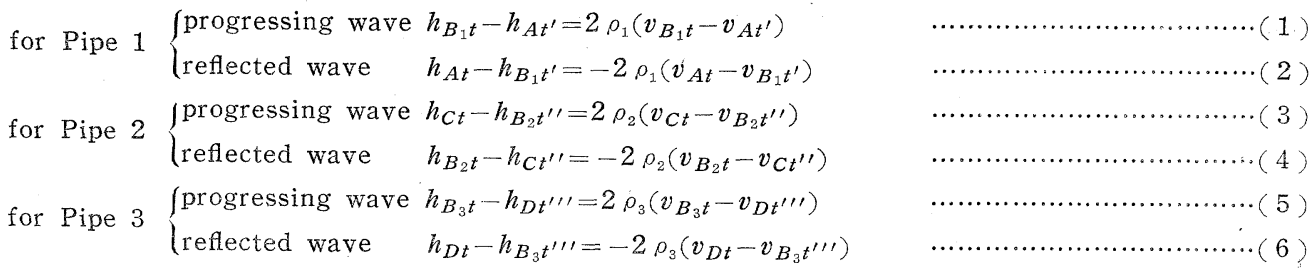

where, denoting the initial state by a bar,

$$
h=H / \vec{H}_{A}, v=A V / A_{1} \bar{V}_{1}, 2 \rho=\alpha \vec{V} / g \vec{H}_{A}=\alpha \vec{Q}_{A} / g \bar{H}_{A} A
$$

and subscripts $t^{\prime}, t^{\prime \prime}$ and $t^{\prime \prime \prime}$ represent the time; for instance, $h_{A t^{\prime}}$ is the head at point $A$ at time $t^{\prime}$.

** Graduate School, Civil Engineering, University of Tokyo. 
Furthermore, the following relationships between time $t$ and $t^{(i)}$ must be satisfied, $t-t^{(i)}=L_{i} / \alpha_{i}$.

The sign of $V_{i}$ is opposit to that of the corresponding wave velocity.

Equation of continuity at the branch point:

$$
A_{2} V_{B_{2}}=A_{1} V_{B_{1}}+A_{3} V_{B_{3}}
$$

or in a non-dimensional form, $\quad v_{B_{2}}=v_{B_{1}}+v_{B_{3}}$

Equal head condition at the branch point:

or

$$
H_{B_{1}}=H_{B_{2}}=H_{B_{3}}+\xi \frac{V_{B_{3}}{ }^{2}}{2 g}
$$

$$
\begin{aligned}
h_{B_{1}} & =h_{B_{2}} \ldots \ldots \ldots \\
& =h_{B_{3}}+a v_{B_{3}}{ }^{2}
\end{aligned}
$$

where, $\xi=$ head loss coefficient for a pipe orifice, $\quad a=\left(\xi / 2 g \bar{H}_{A}\right) \cdot\left(\bar{Q}_{A} / A_{3}\right)^{2}$

The boundary condition at the gate (the orifice law):

$$
V_{A}=\Phi \sqrt{H_{A}} \quad \text { or } \quad v_{A}=\varphi \sqrt{h_{A}}
$$

(b) Graphical solution method*)

There is no difficulty at all in determining the conditions at point $A, C$ or $D$ from those at points $B_{1}, B_{2}$ and $B_{3}$, respectively, by means of an ordinary method. Therefore, the following discussion will be confined to the method for determining the conditions at the branch point.

Equations (1), (4), (5), ( $\left.7^{\prime}\right),(8)$ and $\left(8^{\prime}\right)$ must be solved to determine the unknowns $h_{B_{1} t}=h_{B_{2} t}, h_{B_{3} t}$, $v_{B_{1} t}, v_{B_{2} t}$ and $v_{B_{3} t}$. The method of solution essentially consists of eliminating the unknowns except $\left(h_{B_{1} t}-h_{B_{3}}\right)$ and $v_{B_{3} t}$, from the equations and obtaining a linear equation to be solved simultaneously with the quadratic Equation $\left(8^{\prime}\right)$.

Subtraction of Equation (1) from (4) by using Equations ( $7^{\prime}$ ) and (8), gives.

or

$$
\left(\frac{1}{2 \rho_{1}} h_{A t^{\prime}}+\frac{1}{2 \rho_{2}} h_{C t^{\prime \prime}}\right)-\left(\frac{1}{2 \rho_{1}}+\frac{1}{2 \rho_{2}}\right) h_{B_{1} t}=v_{B_{3} t}-\left(v_{C t^{\prime \prime}}-v_{A t^{\prime}}\right)
$$

where

$$
h_{B_{1} t}-h^{\prime}=-2 \rho_{12}\left(v_{B_{3} t}-v_{B}^{\prime}\right)
$$

$$
\begin{aligned}
& \frac{1}{2 \rho_{12}}=\frac{1}{2 \rho_{1}}-\frac{1}{2 \rho_{2}}=\frac{\rho_{1}+\rho_{2}}{2 \rho_{1} \rho_{2}} \\
& h^{\prime}=2 \rho_{12}\left(\frac{1}{2 \rho_{1}} h_{A t^{\prime}}+\frac{1}{2 \rho_{2}} h_{C t^{\prime \prime}}\right) \\
& v_{B}{ }^{\prime}=v_{C t^{\prime \prime}}-v_{A t^{\prime}} \\
& \text { ions (5) and (10), we obtain }
\end{aligned}
$$$$
\left(h_{B_{1} t}-h_{B_{3} t}\right)-\left(h^{\prime}-h_{D t^{\prime \prime \prime}}\right)=-2 \rho_{123} v_{B_{3} t}+\left(2 \rho_{12} v_{B}{ }^{\prime}-2 \rho_{3} v_{D t^{\prime \prime \prime}}\right)
$$

where

$$
2 \rho_{123}=2 \rho_{12}+2 \rho_{3}=2 \frac{\rho_{1} \rho_{2}+\rho_{2} \rho_{3}+\rho_{3} \rho_{1}}{\rho_{1}+\rho_{2}}
$$

At the graphical intersection of Equation (12) and the remaining quadratic Equation ( $\left.8^{\prime \prime}\right)$,

$$
h_{B_{1} t}-h_{B_{3} t}=a v^{2} B_{3} t
$$

we obtain the conditions at the branch point.

The procedures of the graphical method which mean to trace the above mentioned mathematical treatments on the graphs are shown in Fig. 2. Fig. 3 illustrates an example of the graphical method solved for the run of Fig. 9. The subscripts represent the nondimensional time measured by the unit of $L_{3} / \alpha_{3}$. For convenience in drawing, the procedures have slightly been modified from those of Fig. 2 .

(c) Transmission coefficient for the case of rapid closure.

The following relationships express the state when the gate closes instantly:

*) The graphical method presented by G. BENINI is not correct. (Fig. 7 and 8 in "Sui fenomeni di colpo d'ariete nell gallerie munite di pozzo piezometrico con luce strozzata" L'ENERGIA ELETTRICA, Vol. XXVII, 1950). It can only be applied as a simple approximate method for the following cases: (a) before the negative waves reflected at the reservoir arrive at the branch and (b) the orifice-area ratio is not so small or (c) a branch pipe length is relatively short compared to that of a penstock. 
Fig. 2 Graphical Solution Procedure to Determine a State at the Branch.

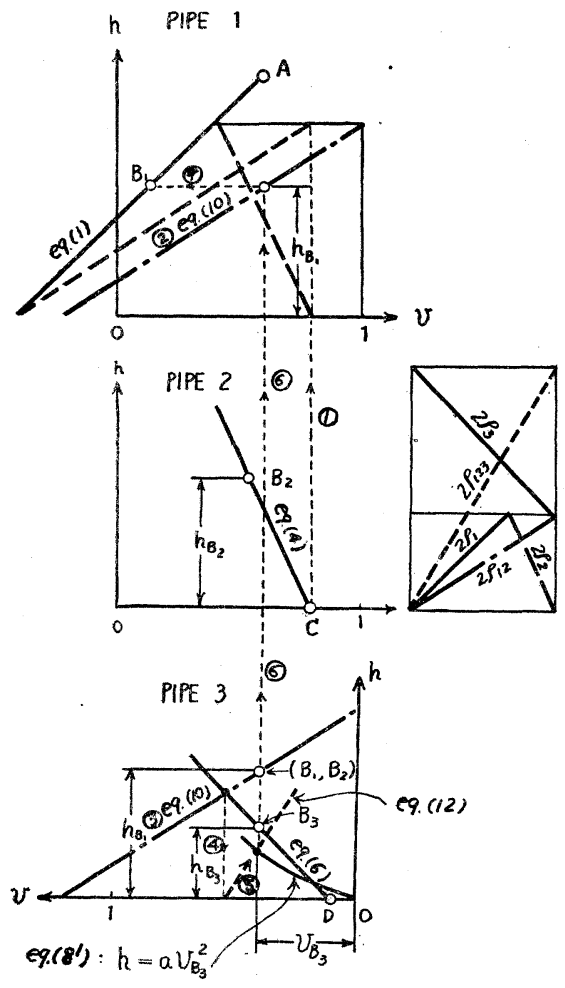

Fig. 3 Example of Graphical Solution Procedure.

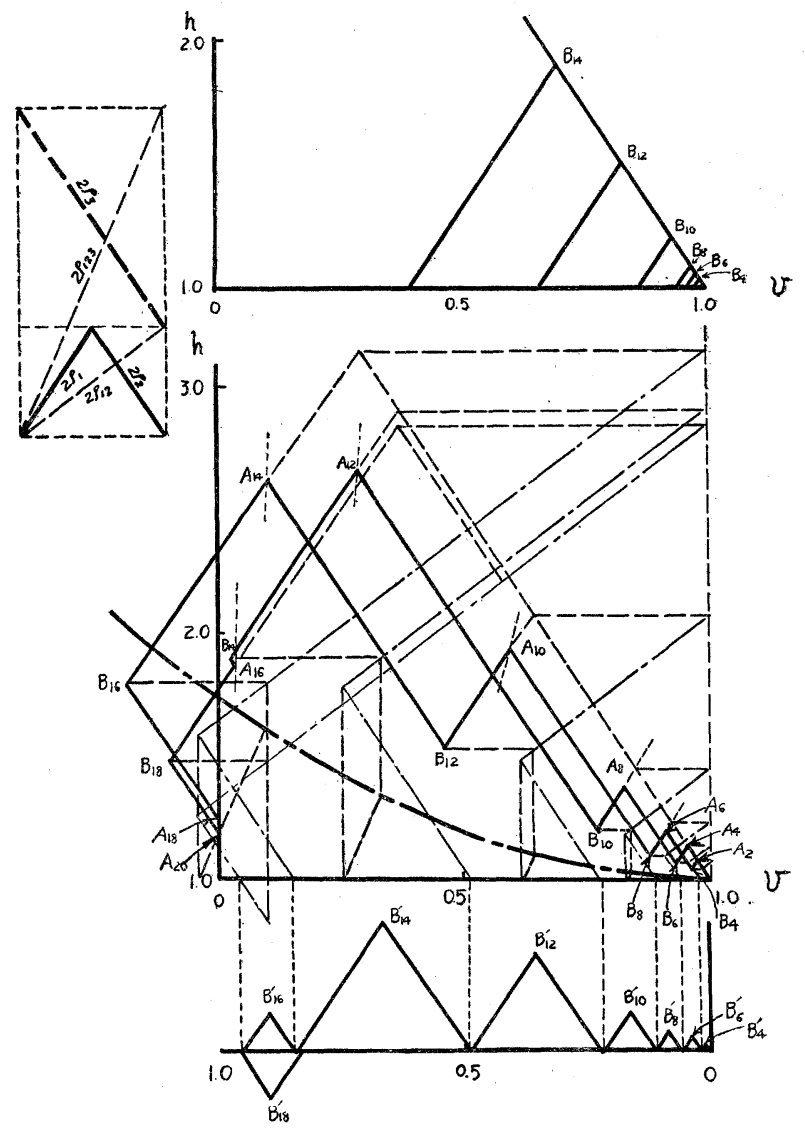

(14)

$$
\left.\begin{array}{l}
v_{D}=0, \quad h_{D}=1, \quad v_{B}{ }^{\prime}=1 \\
h^{\prime}=2 \rho_{12}+1
\end{array}\right\}
$$

Substitution of Equation (14) to Equations (12) and $\left(8^{\prime \prime}\right)$ gives the values of $v_{B_{3} t}$ as follows,

$$
v_{B_{3} t}=\left\{-2 \rho_{123}+\sqrt{\rho_{123}{ }^{2}+4 \rho_{12} a}\right\} / a \text {. }
$$

Therefore, the transmission coefficients $\lambda_{1}$ and $\lambda_{2}$ are

$$
\lambda_{1}=\frac{h_{B_{1}}-1}{h_{A}-1}=\frac{\rho_{12}}{\rho_{1}} \frac{1+\frac{2 a}{\rho_{123}}-\sqrt{1+\frac{4 a \rho_{12}}{\rho_{123}{ }^{2}}}}{\left(\frac{a}{\rho_{123}}\right)}, \cdots \cdots(16) \quad \lambda_{2}=\frac{h_{B_{3}}-1}{h_{A}-1}=\frac{\rho_{3}}{\rho_{1}} \frac{-1+\sqrt{1+\frac{4 a \rho_{12}}{\rho_{123}{ }^{2}}} \cdots}{\left(\frac{a}{\rho_{123}}\right)}
$$

where

$$
\begin{aligned}
& 2 \rho_{1}=\frac{\alpha_{1} \bar{V}_{1}}{g \bar{H}_{A}} \\
& 2 \rho_{12}=\frac{\frac{\alpha_{1} \alpha_{2}{ }^{\prime}}{\alpha_{1}+\alpha_{2}{ }^{\prime}} \bar{V}_{1}}{g \bar{H}_{A}}, \\
& 2 \rho_{123}=\frac{\alpha_{1} \alpha_{2}{ }^{\prime}+\alpha_{2}{ }^{\prime} \alpha_{3}{ }^{\prime}+\alpha_{3}{ }^{\prime} \alpha_{1}}{\alpha_{1}+\alpha_{2}{ }^{\prime}} \frac{\bar{V}_{1}}{g \bar{H}_{A}}, \\
& \alpha_{2}{ }^{\prime}=\frac{A_{1}}{A_{2}} \alpha_{2}, \\
& \alpha_{3}{ }^{\prime}=\frac{A_{1}}{A_{3}} \alpha_{3}, \\
& a=\xi \frac{\bar{V}_{3}{ }^{2}}{2 g \bar{H}_{A}}=\xi \frac{1}{2 g \bar{H}_{A}}\left(\frac{\bar{Q}_{A}}{A_{3}}\right)^{2}
\end{aligned}
$$

For $\alpha_{3}=0$, these relationships result in the formula as proposed in the previous study. ${ }^{1)}$ 
In our experiments, $\alpha_{1}=\alpha_{2}=\alpha_{3}=\alpha, A_{1}=A_{2}=A_{3}=A$ and $2 \rho_{1}=2 \rho_{2}=2 \rho_{3}=2 \rho$. Accordingly, Equations (16) and (17) are simplified further,

$$
\lambda_{1}=\left\{1+\frac{4 a}{3 \rho}-\sqrt{1+\frac{2}{3} \frac{4 a}{3 \rho}}\right\} /\left(\frac{4 a}{3 \rho}\right), \cdots \cdots(19) \quad \lambda_{2}=\left\{-1+\sqrt{1+\frac{2}{3} \frac{4 a}{3 \rho}}\right\} /\left(\frac{1}{2} \cdot \frac{4 a}{3 \rho}\right)
$$

where

$$
\frac{a}{\rho}=\xi \frac{\vec{V}}{\alpha}
$$

\section{EXPERIMENTS :}

(a) Model and Measuring Equipments : The model is composed of a reservoir, a pressure tunnel and a penstock (a steel pipe of $10.5 \mathrm{~cm}$ I.D. by $0.5 \mathrm{~cm}$ thick) a branch pipe acting as a surge tank (the same steel pipe as the main one) and a gate or control valve. The general arrangement of the model is shown in Fig. 4.

\section{Fig. 4 Model of the Branched Pipe System.}
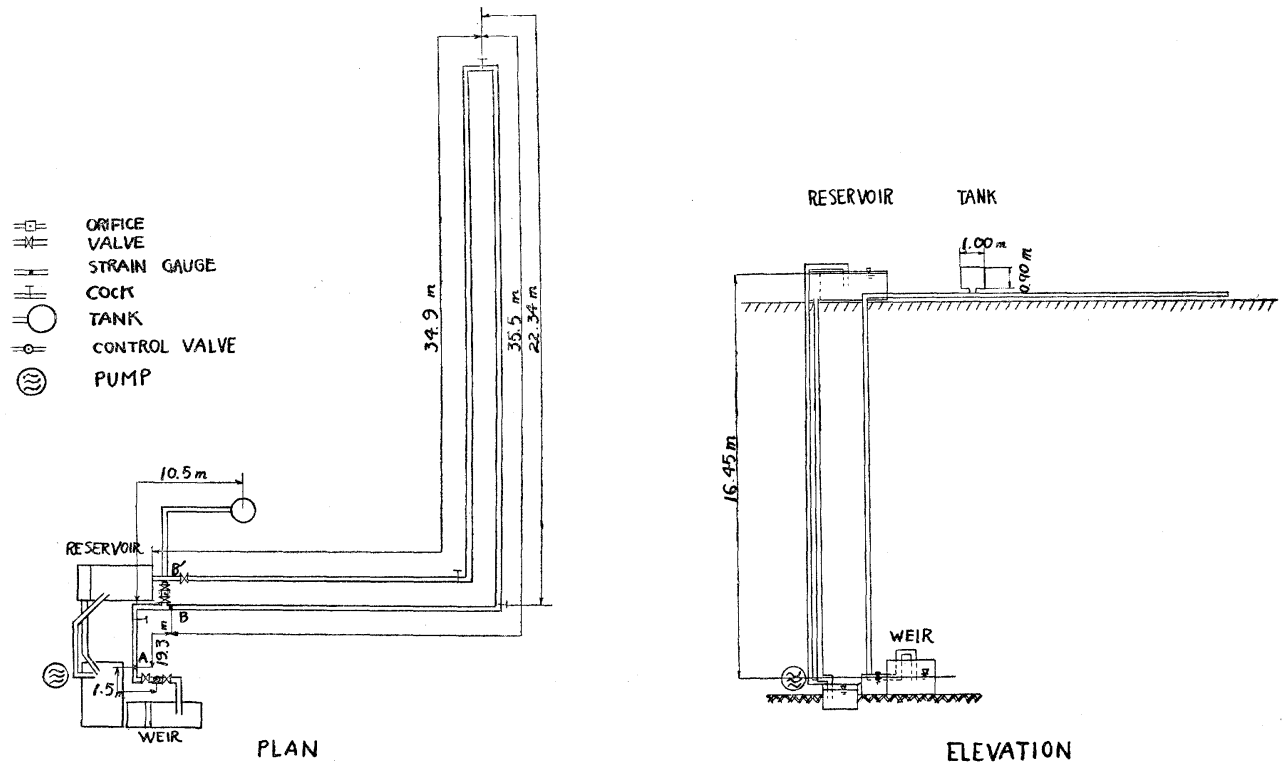

Since a large value of Allievi parameter defined by $2 \rho=\alpha \quad \bar{V} / g \bar{H}$ strengthens the effect of water hammer, a smaller diameter of surge tank is favorable for our purpose. Furthermore, a pipe of sizable length with a simple integer ratio to the length of the main pipes will facillitate the analysis of the experimental results. Consequently, we have simulated the surge tank with a branch pipe.

A branch pipe, $10.5 \mathrm{~m}$ long, was connected with the main one at the distance of $20.8 \mathrm{~m}$ from the gate, where a surge tank had been installed in the previous researches, while at the other end a large (100 cm I.D.) tank was installed to satisfy the boundary condition of constant water level.

Specification of the orifices inserted at the base of the branch pipe are shown in Table 1 . The cross-sectional area of the pipe is denoted by $A_{0}$, and that of each orifice by $A_{p}$.

Table 1

\begin{tabular}{c||c|c|c|c|c|c}
\hline Orifice Number & 1 & 2 & 3 & 4 & 5 & 6 \\
\hline diameter $(\mathrm{cm})$ & 0 & 0.4 & 1.0 & 2.0 & 4.0 & 10.5 \\
\hline$A_{p} / A_{\circ}$ & 0 & $1.45 \times 10^{-3}$ & $9.07 \times 10^{-3}$ & $3.63 \times 10^{-2}$ & $1.45 \times 10^{-1}$ & 1.00 \\
\hline
\end{tabular}

The measuring equipments and experimental procedures were identical to those of the previous study.

Experiments were carried out at a uniform discharge rate of 3 litter/sec. The observed wave velocity ranged from $1370 \mathrm{~m} / \mathrm{s}$ to $1400 \mathrm{~m} / \mathrm{s}$. Three units of pressure pickup (wire strain gages) were installed 
at points $A$ ( $150 \mathrm{~cm}$ upstream from the control valve), $B$ and $B^{\prime}$ (about $30 \mathrm{~cm}$ up- or down-stream from the orifice, respectively). (b) Transmission coefficients : The resistance coefficient of orifice for an unsteady flow differs slightly from those of a steady one, especially when the orifice-area ratio becomes small. However, from the engineering point of view, it is permissible to assume the coefficients for both flows to be equal.4)5) Therefore, the empirical resistance formula derived by OKI, eq. $(22)^{6}$, is sufficient for our purpose.

$$
\xi=\left(\frac{A_{0}}{A_{p}}-1\right)\left(2.75 \frac{A_{0}}{A_{p}}-1.56\right) .
$$

In order to estimate the head loss at the orifice the formula has been extrapolated to Orifices Nos. 2 and 3 beyond the range of applicability, since the smallest value of the orifice-area ratio $A_{p} / A_{0}$ in the experiments from which the OKI formula

Fig. 5 Characteristics of Gate. GATE RATIO

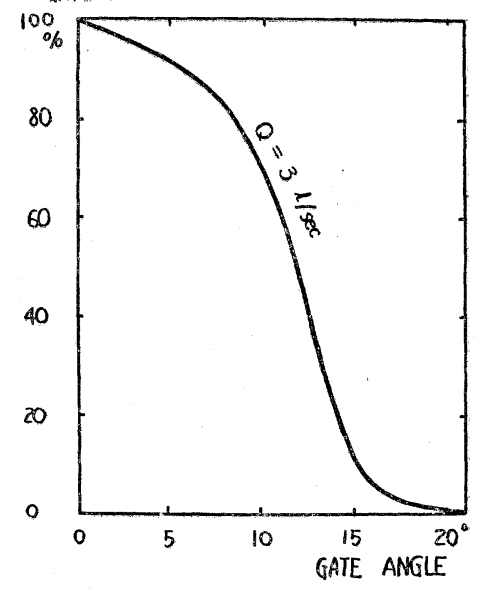
was derived is about 0.05 .

The low limit or minimun value of orifice resistance is given by the nozzle (coefficient of contraction of vena contracta $=1$ ) law, eq. (23).

$$
\xi=\left(\frac{A_{0}}{A_{p}}-1\right)^{2} \text {. }
$$

Only head loss of orifice has been taken into consideration.

Head losses due to bends and variation of pipe section were neglected, because they are small compared to the loss of orifice, especially when the orifice-area ratio becomes small.

First of all,researches on the transmission coefficients for the case of rapid closure of gate were carried out. Comparisons between the results of experiment (each plot is an average of two or three experimental values) and theory, equations (19) (20) and (21), are given in Fig. 6. Experimental points plotted in this

Fig. 6 Transmission Coefficients Theoretical and Experimental; for the Case of Rapid Closure of Gate.

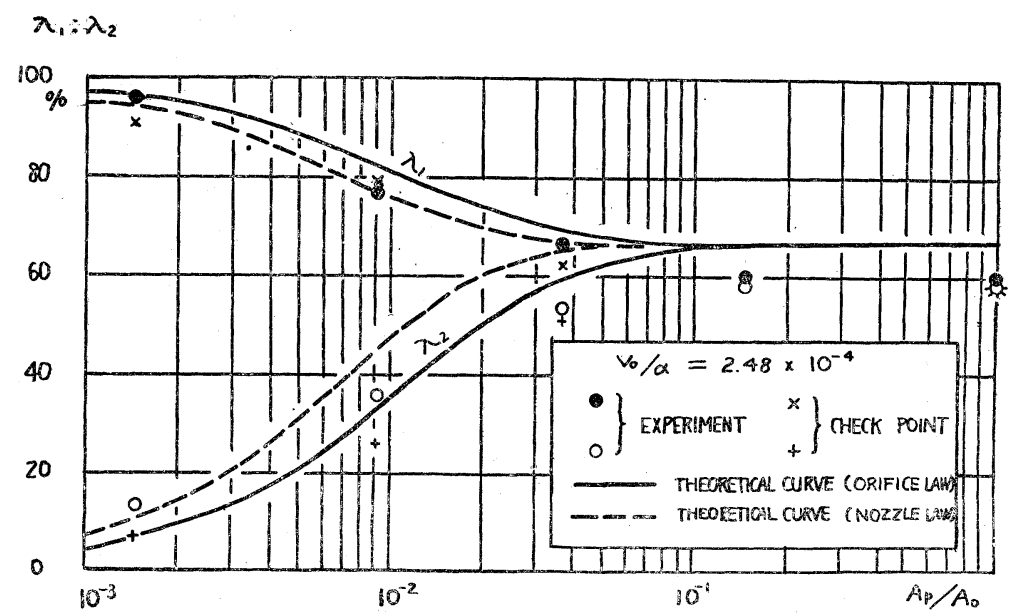

figure deviate considerable from the theoretical curves. This disagreement between experiments and theory can be explained as follows : The theory assumes the rapid closure of gate, that is, the water-hammer wave initiated at the gate at the instant of perfect closure must reach the branch point before the arrival of the reflected initial wave front, or $\left(L_{1} / \alpha_{1}\right)+2\left(L_{3} / \alpha_{3}\right) \geq T+\left(L_{1} / \alpha_{1}\right)$. The gate must be closed perfectly within the time of about $0.015 \mathrm{sec}$ to fulfill this restriction. On the other hand the actual closure time in these experiments ranged from 0.04 to $0.05 \mathrm{sec}$ and can no longer be regarded as rapid closure. The restriction is far severe compared to the one in the previous study (where $T \leqq L_{1} / \alpha_{1}=0.03 \mathrm{sec}$ ). The transmission 
hamax

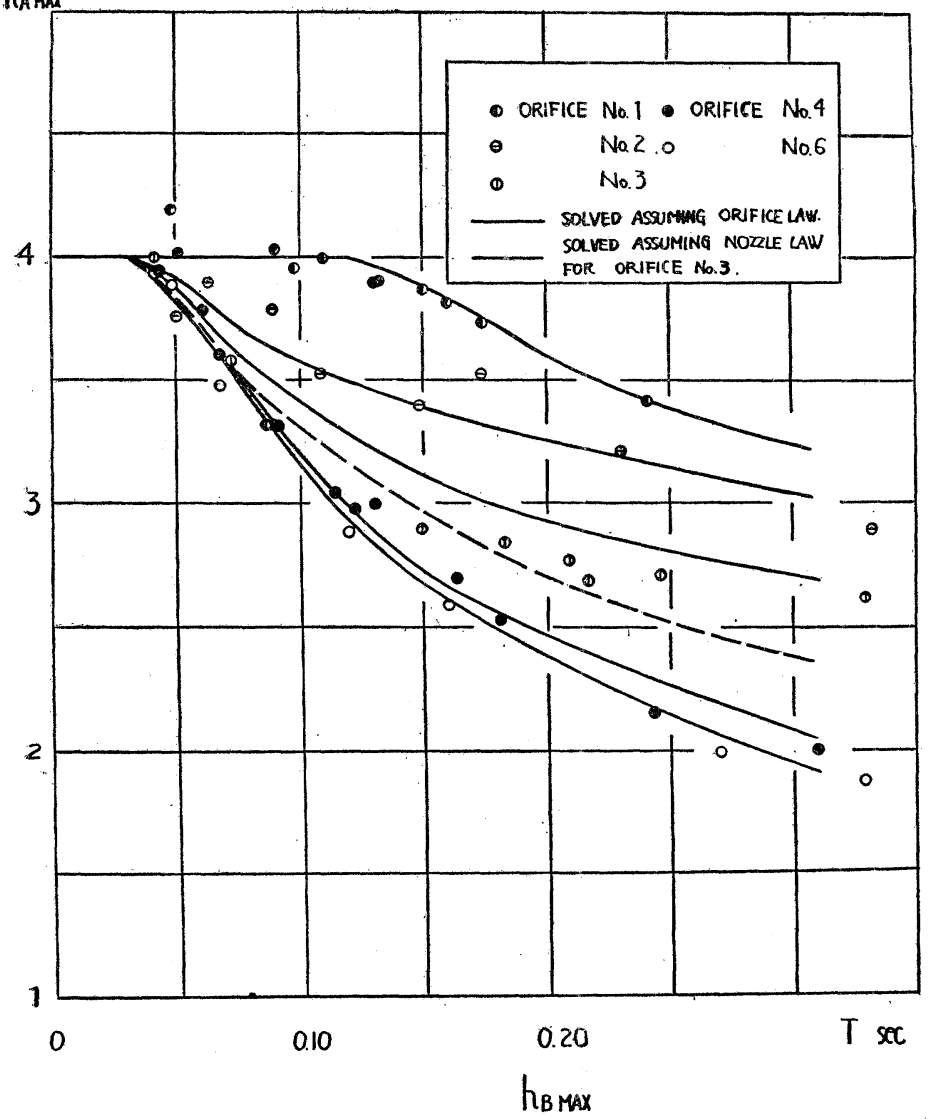

Fig. 7 (a) Relations Between Max Water Hammer Pressures and Closing Time. (Point A)
Fig. 7 (b) Relations Between Max Water Hammer Pressures and Closing Time. (Point B)

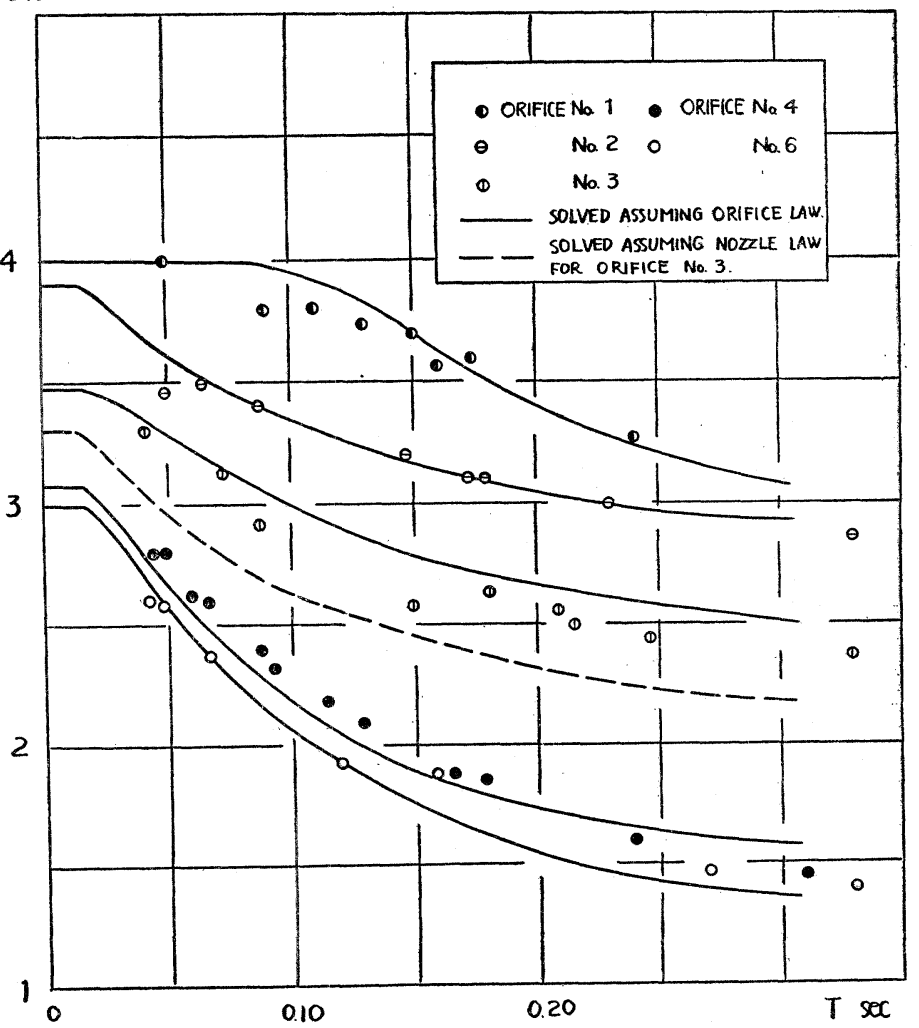


Fig. 7 (c) Relations Between Max Water Hammer Pressures $h_{B^{\prime} \text { MAX }}$ and closing Time. (Point $\mathbf{B}^{\prime}$ )

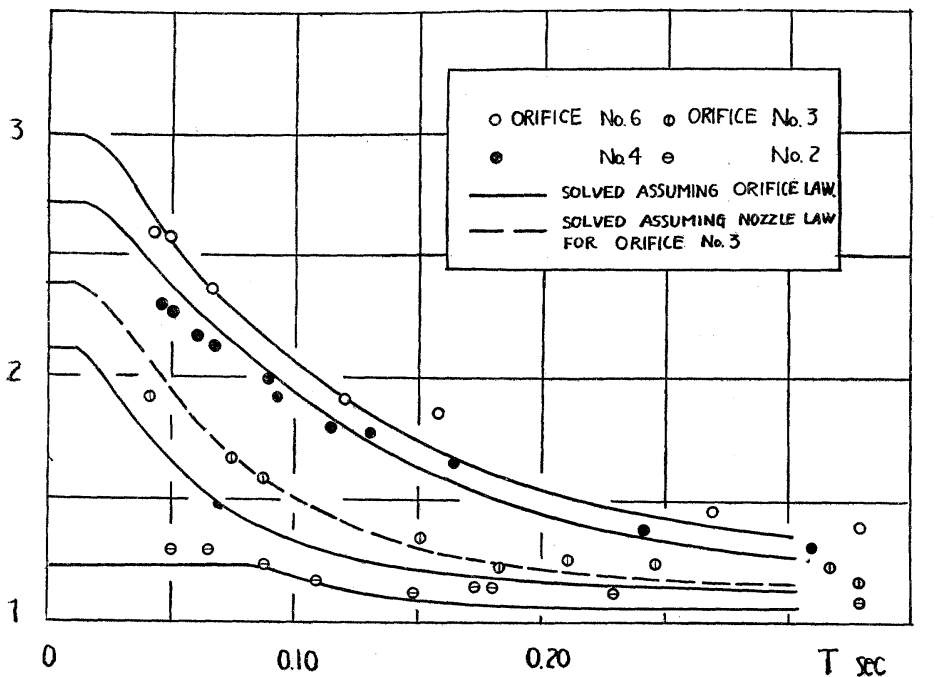

coefficients for the closure time of $0.04 \mathrm{sec}$ were determined through the graghical method, assuming the orifice resistance law, ep. (22), and plotted in Fig. 6 as check points to verify the reason mentioned above. Another reason for the disagreement is the fact that the resistance coefficients of the pipe orifice for accelerated flows is appreciably smaller than for the equivalent steady state ${ }^{4)}$. The mechanical vibration of the pipe caused by a high impulsive pressure rise at point $B$ affected the head at the point $B^{\prime}$ for smaller section of orifice. Therefore, the ex serimental values of $\lambda_{2}$ show a tendency to deviate from the theoretical values as the orifice area decreased. It is note-worthy that the critical ratio of $A_{p} / A_{0}$, below which the transmission coefficients change suddenly, is the same as that found in the previous study. ${ }^{1}$

(c) Closure time and reduction in pressure rise : As the next step, the maximum pressure rise produced by the uniform gradual closure of gate (with a constant angular velocity) have been examined. Results are given in Fig. 7 together with theoretical curves obtained through the graphical method, where the orifice resistance formula, Eq. (22), has been used. The solution based on the nozzle law, eq. (23),

Fig. 8 Comparison Between Theoretical and Experimental Water Hammer Wave. $Q_{0}=3 l \mathrm{~s}, T=0.043 \mathrm{sec}, \phi=10.5 \mathrm{~cm}$

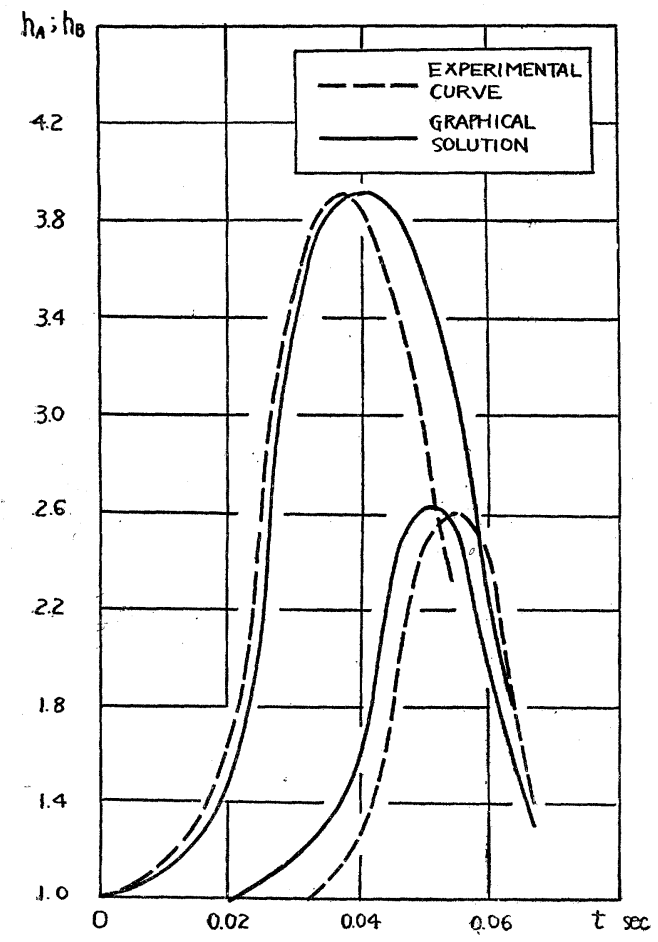

is also shown for Orifice No. 3 by a dotted line.

Agreement between the results of experiment and theory is fair for the simple cases of Orifice No. 1 (simple conduit, $A_{p} / A_{0}=0$ ) and Orifice No. 6 (branched pipe with no restricted orifice, $A_{p} / A_{0}=$ 1). In these two limiting cases, the wave forms are simple as illustrated in Fig. 8. As the orifice comes to play an important role, the wave forms become more complicated. Experimental points for Orifice No. 3 which lie in the centre of the transitional range of Fig. 6 scatter between the two theoretical curves. The exact resistance coefficient of the orifice for unsteady state should be checked by experiment, since, the coefficient assumed for solution has been estimated from extrapolation of the empirical formula for a steady flow beyond the range of experiments from which the formula had been derived.

Fig. 9 and 10 are also examples of water hammer waves theoretical and experimental. The disagreement between the wave forms of experiments and theory is explained as follows:(i)the irregular reflection of the waves from the entraped air in the pipe, (ii) the separation of the air disolved in water at the instant of negative pressure 
(the surcharge head being only 1.2 $m$ for the model). We are now planning to eliminate these disadvantages by removing the pipe line from the roof to the ground level (the surcharge head being $16.45 \mathrm{~m}$ ). (iii) neglect of loss due to bend and (iv) utilization of the orifice resistance coefficient for the turbulent flow even in the laminar zone.

Fig. 11 gives the relationship between the transmission coefficient and the closure time of gate.

\section{CONCLUSIONS :}

From the study on the waterhammer in the branched pipe system with a restricted orifice at the base of the branch, the following conclusions are made.

(a) The transmission coefficients change rapidly as the orifice-area ratio $A_{p} / A_{0}$ is reduced below a certain value which is determined from plotting of eqs. (16), (17) and (18) or eqs. (19), (20) and (21). (b) The larger the orifice area, the greater the decrease of the maximum pressure rise according to the change in closure time since the negative wave reflected at the open end of the branch pipe acts to cancel the positive progressive wave produced at the gate.

(c) The effect of the branch pipe length has not been examined explicitly. However, for the same reason given above it is not difficult to infer that the reduction of pressure rise becomes more remarkable for the shorter branch length.

(d) As the orifice-area ratio is reduced below a critical value or the time of closure is lengthened, pressure rises at $B^{\prime}\left(B_{3}\right)$ decrease nearly to zero, i.e., the effect of
Fig. 9 (a) Comparison Between Theoretical and Exderimental Water Hammer Wave.

$\left(Q_{0}=3 \mathrm{l} / \mathrm{s}, T=0.163 \mathrm{sec}, \phi=2.0 \mathrm{~cm}\right)$

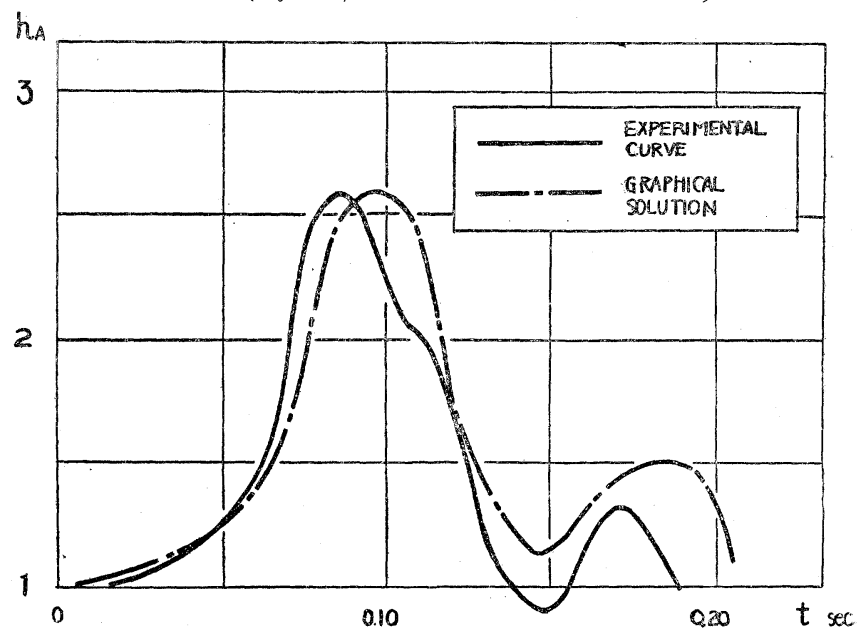

Fig. 9 (b)

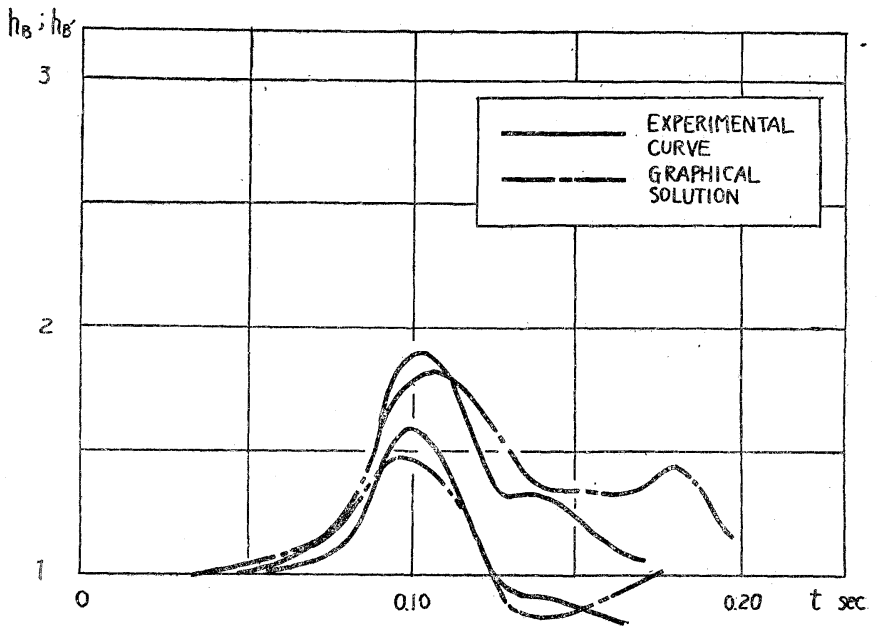

Fig. 10 (a) Comparison Between Theoretical and Experimental Water Hammer Wave.

$\left(Q_{0}=3 \mathrm{l} / \mathrm{s}, T=0.235 \mathrm{sec}, \phi=1.0 \mathrm{~cm}\right)$

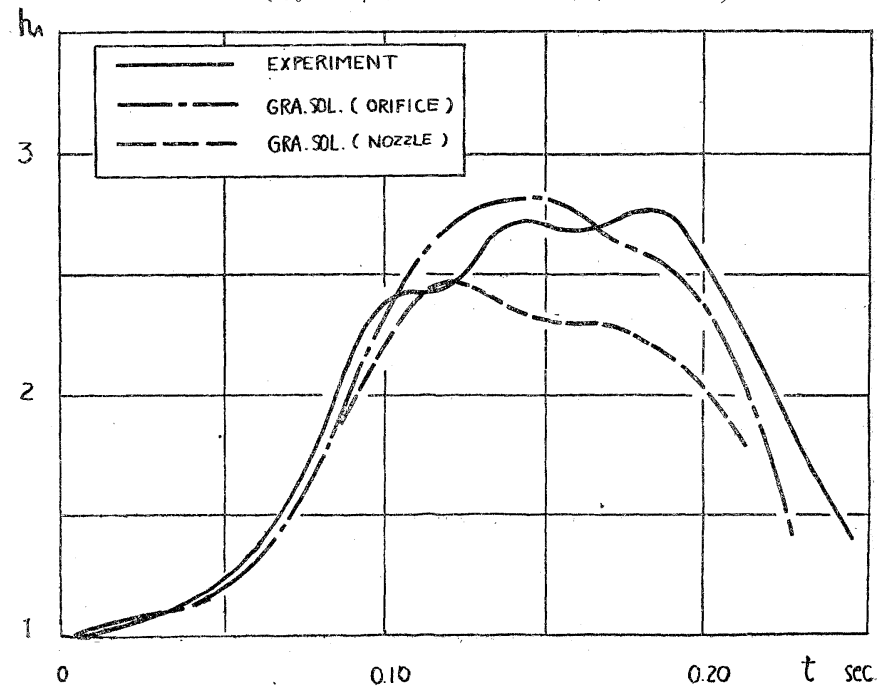


water hammer produced in a branch pipe becomes negligible. (Fig. 7 (c))

Therefore, a branch pipe with small orifice-area ratio can be treated as a simple surge tank, the complexities of analytical procedures can greatly be eliminated.

(e) The reliability of the graphical solution method is satisfactory for engineering purposes.

Since the water hammer depends largely on the characteristics of the gate, pipe line length and area, etc., a strict restriction must be imposed upon derivation of any quantitative properties or formula on the relations between $T$ and $\lambda$, and $T$ and $h_{\max }$ from the results presented in this paper. They must be referred to only to estimate some qualitative tendencies.

\section{Acknowlegements :}

The research was initi-
Fig. $10($ b)

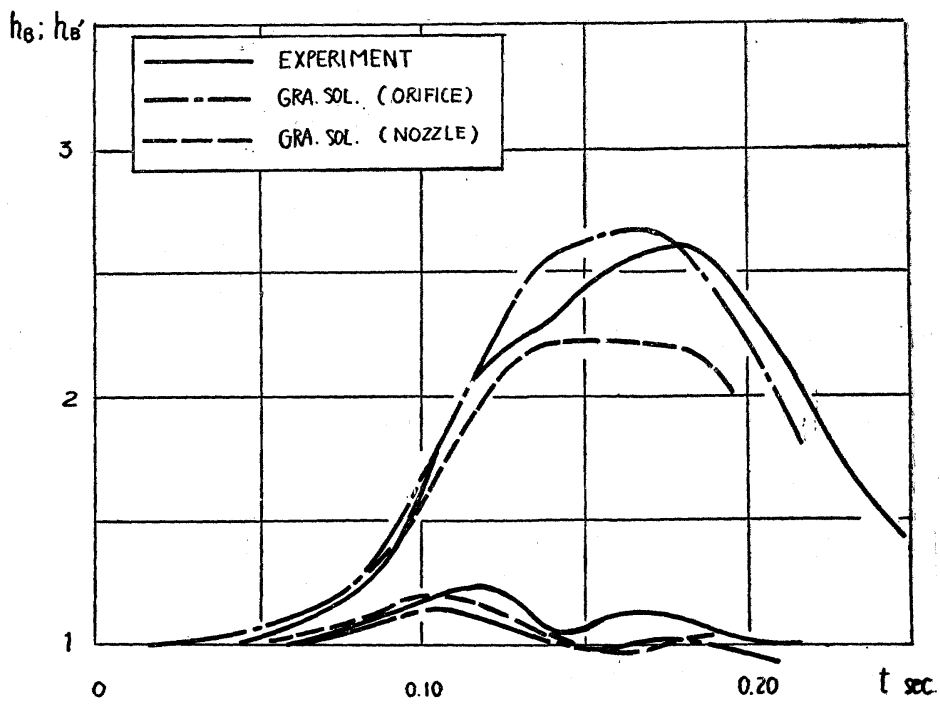

Fig. 11 Relation Between Transmission Coefficient and Closing Time.

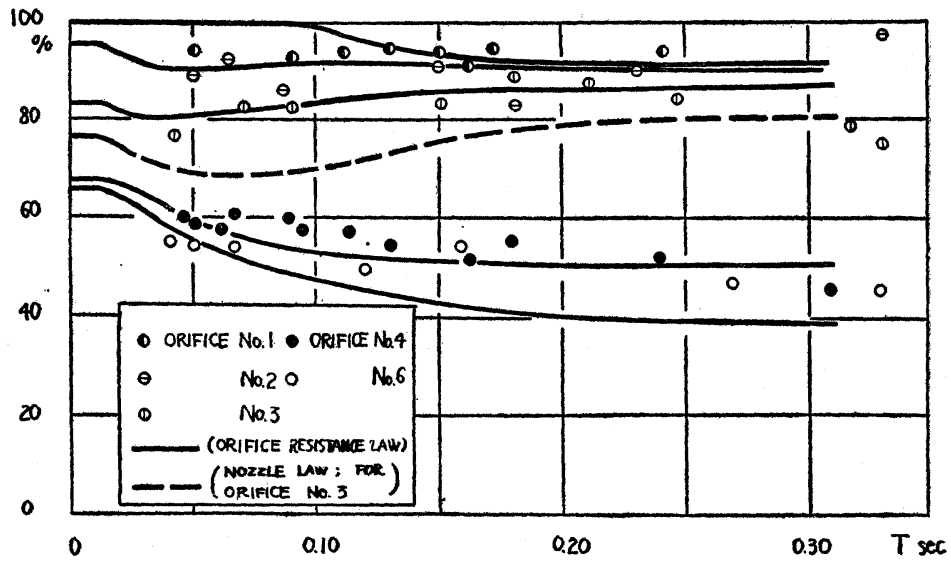
ated by the suggestion of Professor Masashi HOM-MA, University of Tokyo, Japan. The authors wish to express their deep appreciation to Messrs. Yusuke HIROSAWA, research assistant, and Saburo SAIKI, then student of Civil Engineering, University of Tokyo, who participated in the experiments.

\section{References :}

1) SHIMA S. and HINO M. : An experimental study on the effect of restricted orifice surge tank. Trans. JSCE. Vol. 59. (1958)

2) RICH G. : Hydraulic Transients. (1951)

3) ANGUS R.W. : Water hammer pressures in compound and branched pipes, Trans. ASCE Vol. 104 (1939)

4) DAILY J.W., HANKEY W.L., Jr., OLIVE R.W., and JORDAN J.M., Jr., : Resistance coefficients for accelerated and decelerated flows through small tubes and orifices. Trans. ASME, Vol. 78 No. 5 (1956)

5) CARTENS M.R. and ROULLER J.E. : Boundary-shear stress in unsteady turbulent pipe flow. Proc. ASCE HY 2 Vol. 85 (1959)

6) OKI I. : Formulation of the discharge and resistance coefficients for pipe orifices and nozzles. (written in Japanese) Trans. JSME Vol. 4, No. 14 (1938)

\section{CORRIGENDA}

for "An Experimental Study on the Effect of Restricted Orifice Sirge Tank" by S. SHIMA and M. HINO. Trans. JSCE Vol. 59 (1958)

page 61 , line 15; The expression for the term of " $2 \rho_{12}$ " should be changed so that term " $\frac{\alpha_{1} \alpha_{2}}{\alpha_{1}+\alpha_{2}}$ is replaced by " $\frac{\alpha_{1} \alpha_{2}}{\frac{A_{2}}{A_{1}} \alpha_{1}+\alpha_{2}}$ ". 